DrAft VERSION ApriL 6, 2020

Typeset using IATEX RNAAS style in AASTeX63

\title{
In-situ observations of the sub-Alfvénic solar wind by Helios
}

\author{
David Stansby ${ }^{1}$ \\ ${ }^{1}$ Mullard Space Science Laboratory, University College London, Holmbury St. Mary, Surrey RH5 6NT, UK
}

NASA's Parker Solar Probe (PSP, Fox et al. 2016) launched in August 2018, and broke the record for closest spacecraft to the Sun in October 2018. One of the key goals of PSP is to sample the solar wind where the solar wind speed is less than the Alfvén speed. As of the first three orbits this has yet to happen, but as the perihelion distance gradually decreases it is likely to happen in the next couple of years.

PSP is building on the discoveries of the Helios mission, which operated from 1974-1984 at $0.3-1$ AU (Schwenn \& Marsch 1990). Although it did not go as close to the Sun as PSP, Helios did briefly measure sub-Alfvénic solar wind. This finding was presented in the conference proceedings of the Solar Wind Five conference (Neugebauer 1983), but not subsequently published elsewhere. A link to a scanned copy of the proceedings is given below in the references, with the original finding by Schwenn (1983) on pages $502-504$. In this research note a brief overview of the interval is given, along with a summary of the data available for anyone interested in further investigation.

Figure 1 shows an overview of a 4 day interval observed by Helios 1 at distances $0.35-0.39$ AU. The top panel shows the solar wind (blue) and Alfvén (green) speeds. There are two intervals where the wind was clearly sub-Alfvénic: 4 hours starting at the beginning of 1980-06-06, and the second half of 1980-06-07. The wind speed was relatively slow

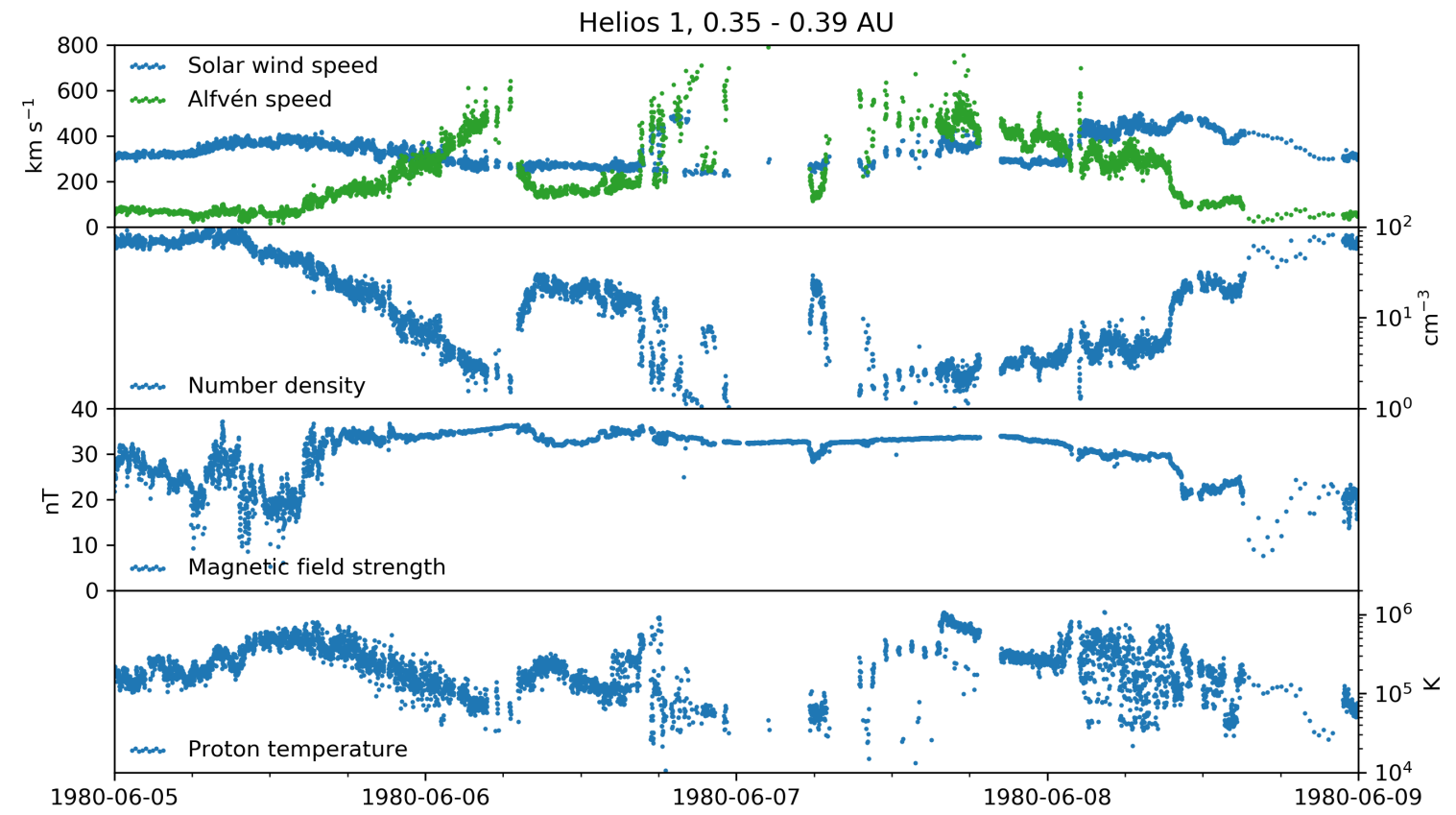

Figure 1. LH column: predicted radial magnetic field at $2.5 R_{\odot}$ (red/blue colourmap) with polarity inversion line (green line) and projected PSP trajectory (black line). Bottom panel shows a zoom with PSP location at 00:00 UTC on each day (black crosses), PSP trajectory (dashed black line), and traced field lines (black lines). RH column: predicted solar wind speed (top panel) and magnetic field polarity (bottom panel). 
$(\mathrm{v} \approx 300 \mathrm{~km} / \mathrm{s}$ ), and the magnetic field magnitude (second panel) relatively constant at around $30 \mathrm{nT}$. A dramatic reduction in the proton number density (third panel) from $50 \mathrm{~cm}^{-3}$ to $2 \mathrm{~cm}^{-3}$ took place just before the first interval, causing a large increase in the Alfvén speed from $50 \mathrm{~km} / \mathrm{s}$ to $400-500 \mathrm{~km} / \mathrm{s}$. The proton temperature (bottom panel) did not show any simultaneous significant differences compared to nearby examples of super-Alfvénic wind.

For the interval shown several datasets are available: plasma moments (used here), proton fits (containing velocity vectors, and parallel and perpendicular temperatures), positive ion 3D distribution functions, and electron 2D distribution functions all at 40.5 second cadence, and vector magnetic field data at 6 second cadence. This data is all available on the Helios Data Archive (http://helios-data.ssl.berkeley.edu).

A shorter sub-Alfvénic was also observed by Helios 2 on 1979-11-13, but in this case the solar wind was so slow $(<170$ $\mathrm{km} / \mathrm{s}$ ) as to drop out of the detecting range of the plasma instrument (Schwenn 1983). This event was accompanied by a similar sub-Alfvénic at the near-Earth solar wind, documented by Gosling et al. (1982). The lack of subsequent observations these low density events points to their rarity and transient nature, but their relatively short duration leaves little data to investigate the properties of sub-Alfvénic wind. As PSP lowers its perihelion in the coming years, hopefully it will spend a significant amount of time in sub-Alfvénic wind and, with it's improved instrumentation, provide new insight into the mechanisms that heat and accelerate the solar wind.

Software: HelioPy 0.10.1 (Stansby et al. 2020), Matplotlib 3.2.1 (Hunter 2007; Caswell et al. 2020)

\section{REFERENCES}

Caswell, T. A., Droettboom, M., Lee, A., et al. 2020, Matplotlib/Matplotlib: REL: V3.2.1, Zenodo.

https://doi.org/10.5281/zenodo.3714460

Fox, N. J., Velli, M. C., Bale, S. D., et al. 2016, Space

Science Reviews, 204, 7, doi: 10.1007/s11214-015-0211-6

Gosling, J. T., Asbridge, J. R., Bame, S. J., et al. 1982, Journal of Geophysical Research: Space Physics, 87, 239, doi: 10.1029/JA087iA01p00239

Hunter, J. D. 2007, Computing in Science \& Engineering, 9, 90, doi: 10.1109/MCSE.2007.55
Neugebauer, M. 1983, Solar Wind Five (NASA Conference Report). https://ntrs.nasa.gov/search.jsp?R=19840004999

Schwenn, R. 1983, in Solar Wind Five, Woodstock, Vermont, 489-504

Schwenn, R., \& Marsch, E., eds. 1990, Physics of the Inner Heliosphere I: Large-Scale Phenomena, Physics and Chemistry in Space (Berlin Heidelberg: Springer-Verlag). https://www.springer.com/us/book/9783642753633

Stansby, D., Rai, Y., Argall, M., et al. 2020, Heliopython/Heliopy: HelioPy 0.10.1, Zenodo. https://doi.org/10.5281/zenodo.3739114 